\title{
EMBEDDING GRAPHS INTO COLORED GRAPHS
}

\author{
A. HAJNAL AND P. KOMJÁTH
}

\begin{abstract}
If $X$ is a graph, $\kappa$ a cardinal, then there is a graph $Y$ such that if the vertex set of $Y$ is $\kappa$-colored, then there exists a monocolored induced copy of $X$; moreover, if $X$ does not contain a complete graph on $\alpha$ vertices, neither does $Y$. This may not be true, if we exclude noncomplete graphs as subgraphs.

It is consistent that there exists a graph $X$ such that for every graph $Y$ there is a two-coloring of the edges of $Y$ such that there is no monocolored induced copy of $X$. Similarly, a triangle-free $X$ may exist such that every $Y$ must contain an infinite complete graph, assuming that coloring $Y$ 's edges with countably many colors a monocolored copy of $X$ always exists.
\end{abstract}

0. Introduction. In this paper we deal with the generalization of partition theory which investigates the existence of monocolored prescribed subgraphs of multicolored graphs satisfying certain conditions. As usual we will need partition symbols to make the formulation of the results and problems feasible.

(0.1) $Y \rightarrow(X)_{\gamma}^{1}, Y \rightarrow(X)_{\gamma}^{2}$ mean that the following statements are true. If the vertices/edges of $Y$ are $\gamma$-colored then there exists a monocolored copy of $X \subset Y$, respectively.

(0.2) $Y \hookrightarrow(X)_{\gamma}^{1}, Y \mapsto(X)_{\gamma}^{2}$ mean the existence of monocolored copies of $X$ which are induced subgraphs of $Y$.

Clearly, the Erdös-Rado generalization of Ramsey's theorem yields an obvious existence theorem of type $\forall X \exists Y$ in (0.1), and the meaningful results concerning this symbol are of the form $\forall X \in \mathscr{G} \exists Y \in \mathscr{E}$ for certain classes $\mathscr{G}, \mathscr{E}$ of graphs.

The existence problem for the symbols $(0.2)$ is nontrivial, though it is quite easy for the first symbol and here the problem has to be investigated under additional restrictions on $X$ and $Y$. As to the symbol $Y \mapsto(X)_{\gamma}^{2}$ the statement

$$
\forall X \exists Y \quad Y \mapsto(X)_{\gamma}^{2} \text { for } \gamma<\omega, X \text { (and } Y \text { ) finite }
$$

was proved by three different sets of authors $[\mathbf{4}, \mathbf{1 0}, \mathbf{2 0}]$ and it was extended for countable graphs $X$ in $[\mathbf{1 0}]$ where $\forall \gamma<\omega \forall|X| \leq \omega \exists|Y| \leq 2^{\omega} Y \mapsto(X)_{\gamma}^{2}$ was proved.

One of the main observations of this paper is that (contrary to the intuitive expectation of combinatorialists that this kind of Ramsey property always holds

Received by the editors March 21, 1986 and, in revised form, April 15, 1987.

1980 Mathematics Subject Classification (1985 Revision). Primary 03E05; Secondary 03E35, 04A20, $05 \mathrm{C} 65$.

Key words and phrases. Infinite graphs, Ramsey theory, independence, forcing, hypergraphs, chromatic number.

Research partially suppported by the Hungarian National Foundation for Scientific Research Grant \#1805. 
with a sufficiently large $Y)$ it is consistent that

(0.3) $\exists X|X|=\omega_{1} \forall Y Y \nrightarrow(X)_{2}^{2}$ holds.

This is a corollary of our more technical Theorem 12 . Moreover $(0.3)$ holds in a very simple model (adding one Cohen real) which does not influence the existence of large cardinals.

This leaves the following problem open.

(0.4) Is it true that for all countable $X$ there is a $Y$ with $Y \mapsto(X)_{\omega}^{2}$ ?

We have no guess if the negation of $(0.3)$ is consistent. The most natural assumption to investigate the symbols $(0.1)$ and the first symbol of $(0.2)$ is to assume that both $X$ and $Y$ are graphs not containing $K_{\alpha}$, the complete graph on $\alpha$ vertices. (Note that it is customary to use $K_{\tau}$ only if $\tau$ is a cardinal, but if the underlying set of $X$ and $Y$ has a natural (well)-ordering, $K_{\alpha}$ has a self-explanatory meaning.) Let $\mathcal{N}_{\alpha}$ be the class of graphs not containing $K_{\alpha}$. Folkman [12] and Nesetril and Rödl [19] worked out the positive results for the finite case, showing

$$
\forall i=1,2 \quad \forall \alpha, \gamma<\omega \quad \forall X \in \mathscr{N}_{\alpha}\left(|X|<\omega \rightarrow \exists Y \in \mathscr{N}_{\alpha}|Y|<\omega Y \rightarrow(X)_{\gamma}^{i}\right)
$$

For infinite $X$ and $\gamma$ but finite $\alpha$ this was only recently proved for $i=1$ by the second author and Rödl [17].

We extend this result for every $\alpha$ (see Theorem 1). The first section analyzes the problems raised by this type of results.

For regular $\kappa \geq \omega,|X|=\kappa$ and $\gamma=\kappa$ our theorem yields a $Y$ of size $2^{\kappa}$ and in Theorem 2 we prove that it is consistent that $2^{\kappa}>\kappa^{+}$and still a $Y$ of size $\kappa^{+}$ always suffice.

This raises the problem if $Y \hookrightarrow(X)_{\gamma}^{1}$ is absolute with respect to certain Cohen extensions. We have several relevant remarks. We state here only the simplest instances. Theorem 3 tells us that if $|Y| \leq 2^{\omega}$ and $\operatorname{Chr}(Y)>\omega$ then this second property of $Y$ cannot be destroyed by adding Cohen reals, and that the assumption $|Y| \leq 2^{\omega}$ is necessary for this statement. In Theorem 4 we show that on the other hand there is a graph $Y$ on $2^{\omega}$ with $\operatorname{Chr}(Y)>\omega$ which can be made $\omega$ chromatic by c.c.c. forcing. On the other hand we prove in this section (Theorem 5) that proper forcing cannot destroy the property of a graph having coloring number greater than $\omega$. Theorem 6 is a strengthening of Theorem 1 under the assumption $V=L$ yielding a strong incompactness result as well.

Finally we consider in this section graphs not containing $K_{\omega}$ i.e. containing only finite complete subgraphs, in more detail. There is an obvious way to define the rank of such graphs by the rank of the well-founded partial order of finite complete subgraphs ordered by reverse inclusion. We restate the result of Theorem 1 in terms of the rank and we prove the existence of universal graphs for graphs having a fixed cardinality $\kappa$ and rank $\leq \alpha<\kappa^{+}$. See Theorem 7 .

In $\S 2$ we prove results of the following type. If $Y \rightarrow(X)_{\omega}^{1}$ or $Y \rightarrow(X)_{\omega}^{2}$ holds then under certain conditions $Y$ must contain something larger than $X$. We need some elaborate finite graph constructions for this purpose which we hope might be of some independent interest.

As a corollary of our results in $\S 1$, for every finite $X$ and $\alpha<\omega, X \in \mathscr{N}_{\alpha}$ there is a $Y \in \mathscr{N}_{\alpha},|Y| \leq 2^{\omega}$ such that $Y \longmapsto(X)_{\omega}^{1}$. In Theorem 9 we show that this very strongly fails for the class $\mathcal{N}_{4}^{-}$, the set of graphs not containing $K_{4}^{-}$(the graph $K_{4}$ 
minus one edge); namely for every $n=1,2, \ldots$ there are $T_{n} \in \mathscr{N}_{4}^{-}$such that for all $Y \in \mathscr{N}_{4}^{-}$with $Y \rightarrow\left(T_{n}\right)_{\omega}^{1},|Y|>\omega_{n}$.

Moreover we show in Theorem 10 that there is a countable $X \in \mathscr{N}_{4}^{-}$such that $Y \rightarrow(X)_{\omega}^{1}$ holds for no $Y \in \mathcal{N}_{4}^{-}$.

In $\S 3$ using a technique of Shelah we first prove a stronger form of $(0.3)$, showing that the existence of a nonarrowable bipartite $X$ on the vertex set $\omega \cup \omega_{1}$ is consistent.

The next theorem is also a strengthening of (0.3) at least for countably many colors. Namely it tells us that it is consistent to have a triangle-free $X$ of size $\omega_{1}$ such that for all $Y$ with $Y \rightarrow(X)_{\omega}^{2} Y$ contains a $K_{\omega}$, and the proof indeed yields that for all $Y$ there is an edge coloring $f$ so that for every monochromatic embedding $g$ of $X$ into $Y g^{\prime \prime} X$ contains a $K_{\omega}$, hence $Y \nrightarrow(X)_{\omega}^{2}$ holds for all $Y$ and this $X$.

We do not know if $\omega$ can be replaced by 2 in this theorem, and we also do not know if $K_{\omega}$ can be replaced by $K_{\omega_{1}}$ i.e.

(0.6) Is it consistent that there exists a triangle-free $X$ of size $\omega_{1}$ such that $K_{\omega_{1}} \subset Y$ holds for every $Y$ with $Y \mapsto(X)_{\omega}^{2}$ (or $\left.Y \mapsto(X)_{2}^{2}\right)$ ?

Note that $K_{\omega_{1}}$ cannot be replaced by $K_{\left(2^{\omega}\right)}+$ since as a corollary of the relations

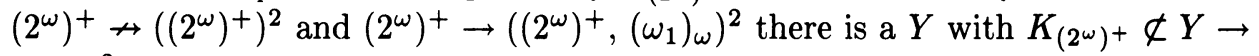
$\left(K_{\omega_{1}}\right)_{\omega}^{2}$.

Finally, Theorem 14 tells us that it is consistent that almost all graphs of size $\omega_{1}$ will satisfy (0.3) at least if we make a restriction on the size of $Y$.

Our notation is standard. $X \leq Y$ denotes that $X$ is isomorphic to a subgraph of $Y,[A]^{\mu},[A]^{<\mu}$ denote $\{x \subseteq A:|x|=\mu\},\{x \subseteq A:|x|<\mu\}$, respectively.

\section{Vertex partitions.}

THEOREM 1. If $X$ is a graph on $\kappa=\operatorname{cf}(\kappa) \geq \omega$, then there exists a graph $Y$ on $2^{\kappa}$ such that $Y \mapsto(X)_{\kappa}^{1}$ and $K_{\alpha} \npreceq Y$ for every $\alpha$ with $K_{\alpha} \npreceq X$.

ProOF. For $\xi<\kappa$ put $X(\xi)=\{\varsigma<\xi:\{\zeta, \xi\} \in X\}$. We are going to construct the sets $Y(\xi)=\{\varsigma<\xi:\{\varsigma, \xi\} \in Y\} \subseteq \xi$ by recursion on $\xi<2^{\kappa}$ and simultaneously assign to every $s \subseteq 2^{\kappa},|s| \geq 2$ with $[s]^{2} \subseteq Y$ a set $\Phi(s) \subseteq \kappa$ with $[\Phi(s)]^{2} \subseteq X$ and satisfying the additional condition

(1.1) if $t$ end-extends $s$, then $\Phi(t)$ (properly) end-extends $\Phi(s)$.

As (1.1) obviously implies $\operatorname{tp}(\Phi(s)) \geq \operatorname{tp}(s)$, if $K_{\alpha} \not \Varangle X, K_{\alpha} \npreceq Y$ is guaranteed.

An embedding is an increasing function $f$ from an ordinal $\delta \leq \kappa, f: \delta \rightarrow 2^{\kappa}$ with the following properties:

(1.2) for $\beta<\gamma<\delta\{\beta, \gamma\} \in X$ iff $\{f(\beta), f(\gamma)\} \in Y$;

(1.3) if $s \subseteq \delta,|s| \geq 2$, and $s$ spans a complete graph in $X, s=\Phi\left(f^{\prime \prime} s\right)$.

$Y$ will be constructed under the following conditions:

(1.4) if for $\gamma<\kappa, f_{\gamma}: \delta_{\gamma} \rightarrow 2^{\kappa}$ are embeddings with $\delta_{\gamma}<\kappa, \operatorname{Rng}\left(f_{\gamma}\right)=A_{\gamma}$, $\sup \left(A_{\gamma}\right)<\inf \left(A_{\gamma^{\prime}}\right)\left(\gamma<\gamma^{\prime}<\kappa\right)$, no edge goes between any two of the $A_{\gamma}$ 's, then there exists a point $\beta>\sup \left(\bigcup\left\{A_{\gamma}: \gamma<\kappa\right\}\right)$ with $Y(\beta)=\bigcup\left\{B_{\gamma}: \gamma<\kappa\right\}$ where $B_{\gamma}=f_{\gamma}^{\prime \prime} X\left(\delta_{\gamma}\right)$ and, if for $\gamma<\kappa, s \subseteq X\left(\delta_{\gamma}\right), s \neq \varnothing,[s]^{2} \subseteq X$, then $\Phi\left(f_{\gamma}^{\prime \prime} s \cup\{\beta\}\right)=s \cup\left\{\delta_{\gamma}\right\}$

(1.5) every $Y(\beta)$ is constructed in a step of type (1.4).

Notice that these conditions imply

(1.6) $\operatorname{tp}(Y(\beta)) \leq \kappa$ for $\beta<2^{\kappa}$. 
We extend $\Phi$ to sets of limit type the obvious way:

(1.7) if $s \subseteq 2^{\kappa}, s \neq \varnothing$, and $s$ has no last element, then

$$
\Phi(s)=\bigcup\{\Phi(s \cap \mu): \mu<\sup (s)\} .
$$

We have to show that $Y \hookrightarrow(X)_{\kappa}^{1}$ holds (the other properties are clear). Assume that $g: 2^{\kappa} \rightarrow \kappa$ is a $\kappa$-coloring with no monocolored induced copy of $X$. An embed$\operatorname{ding} f: \delta \rightarrow 2^{\kappa}$ with $\operatorname{Rng}(g \circ f)$ having exactly one element is called monochromatic, if its unique element is $\mu$, it is $\mu$-colored. $f$ is nonextendable, if it is monochromatic but it has no monochromatic (end) extension.

ClaIM. There exists a $\beta<2^{\kappa}$ such that for every $\gamma$ with $\beta<\gamma<2^{\kappa}$, and for every $\tau<\kappa$, there is a nonextendable $\tau$-colored embedding $f$ with $\operatorname{Rng}(f) \subseteq\left[\gamma, 2^{\kappa}\right)$, with no edge between $[\beta, \gamma)$ and $\operatorname{Rng}(f)$.

PROOF OF THE ClAIM. Assuming the claim is false, we can recursively find $\beta_{\xi}$, $\gamma_{\xi}$, and $\tau_{\xi}$ for $\xi<\kappa^{+}$with

(1.8) $\beta_{\xi}<\gamma_{\xi}<\beta_{\xi^{\prime}}<2^{\kappa}, \tau_{\xi}<\kappa$ for $\xi<\xi^{\prime}<\kappa^{+}$;

(1.9) if $f$ is a nonextendable, $\tau_{\xi}$-colored embedding into $\left[\gamma_{\xi}, 2^{\kappa}\right)$ then there is an edge between $\left[\beta_{\xi}, \gamma_{\xi}\right)$ and $\operatorname{Rng}(f)$.

As $\operatorname{cf}\left(2^{\kappa}\right)>\kappa$, no problem with the construction arises. Choose $\tau<\kappa$ with $|S|=$ $\kappa^{+}$, where $S=\left\{\xi<\kappa^{+}: \tau_{\xi}=\tau\right\}$. Pick the first $\kappa^{2}$ elements of $S:\left\{\delta_{\xi}: \xi<\kappa^{2}\right\}$ (here $\kappa^{2}$ denotes ordinal square). Let $f$ be a nonextendable $\tau$-colored embedding into $\left[\sup \left\{\gamma_{\delta_{\xi}}: \xi<\kappa^{2}\right\}, 2^{\kappa}\right)$, put $B=\operatorname{Rng}(f)$. (Such an $f$ exists by Zorn's lemma.) As no monocolored induced copy of $X$ exists in $Y,|B|<\kappa$. By hypothesis, for $\xi<\kappa^{2}$, there is an edge between $\left[\beta_{\delta_{\xi}}, \gamma_{\delta_{\xi}}\right)$ and $B$. For $b \in B$, the set $\left\{\xi<\kappa^{2}: b\right.$ is joined to $\left.\left[\beta_{\delta_{\xi}}, \gamma_{\delta_{\xi}}\right)\right\}$ has order-type $\leq \kappa$ by (1.5), so $\kappa^{2}$ is the union of $<\kappa$ sets, each of type $\leq \kappa$, which is absurd.

Choose $\beta<2^{\kappa}$ as in the Claim. By induction on $\tau<\kappa$ we choose $\beta_{\tau}<\gamma_{\tau}$, a nonextendable $\tau$-colored embedding $f_{\tau}: \alpha_{\tau} \rightarrow\left[\beta_{\tau}, \gamma_{\tau}\right)$ satisfying

(1.10) $\beta_{0}=\beta, \gamma_{\tau^{\prime}}<\beta_{\tau}$ for $\tau^{\prime}<\tau<\kappa, \alpha_{\tau}<\kappa$

(1.11) no edge goes between $\left[\beta, \beta_{\tau}\right)$ and $\operatorname{Rng}\left(f_{\tau}\right)$.

By (1.4), there is a point $\delta>\sup \left\{\gamma_{\tau}: \tau<\kappa\right\}$ such that $f_{\tau} \cup\left\{\left\langle\alpha_{\tau}, \delta\right\rangle\right\}$ is an embedding for $\tau<\kappa$. This implies that the color of $\delta$ is not $\tau$ for every $\tau<\kappa$, a contradiction.

Next we show that a $Y$ as described in Theorem 1 may exist with $|Y|=\kappa^{+}<2^{\kappa}$.

THEOREM 2. Assume that $V$ models $\kappa^{\kappa}=\kappa, 2^{\kappa}=\kappa^{+}, \operatorname{cf}(\lambda)>\kappa, \lambda^{\kappa}=\lambda$. There exists a generic extension blowing $2^{\kappa}$ up to $\lambda$ such that for every $X$ on $\kappa$ there exists a $Y$ on $\kappa^{+}$such that $Y \mapsto(X)_{\kappa}^{1}$ and $K_{\alpha} \Varangle Y$ whenever $K_{\alpha} \nless X$.

PROOF. The applied notion of forcing is the Cohen partial order making $2^{\kappa}=\lambda$ with $<\kappa$-size conditions. If $X$ is a graph on $\kappa$ in the generic extension, then by the $\kappa^{+}$-c.c. $X$ is in an intermediate model generated by a subset of size $\leq \kappa$ of the original notion of forcing. By the product lemma (see [18]) the final model can be regarded as a generic extension of this model; the extension is done by the same Cohen-forcing blowing $2^{\kappa}$ up to $\lambda$, so we can simply assume that $X \in V$. Let $Y$ be the graph constructed in Theorem 1 (in $V$ ). We show that $Y \mapsto(X)_{\kappa}^{1}$ still holds in the expanded model. That $K_{\alpha} \nless \Varangle Y$ still holds is obvious if $\alpha<\kappa^{+}$by the $<\kappa$-closure property of the notion of forcing. Also, $K_{\kappa^{+}} \nless Y$ holds by (1.6). 
First we show that a suitable form of the Claim in Theorem 1 is true. Let $P$ be the notion of forcing, $p \in P$ and $p \Vdash " g: \kappa^{+} \rightarrow \kappa$ has no monocolored copy of $X$ ".

Claim. $p$ forces that there exists a $\beta<\kappa^{+}$such that for every $\gamma$ with $\beta<$ $\gamma<\kappa^{+}$and for every $\tau<\kappa$ there is a nonextendable $\tau$-colored embedding $f$ with $\operatorname{Rng}(f) \subseteq\left[\gamma, 2^{\kappa}\right)$, with no edge between $[\beta, \gamma)$ and $\operatorname{Rng}(f)$.

PROOF OF THE CLAIM. If $p$ does not force the statement, we can recursively find $\beta_{\xi}, \gamma_{\xi}, \tau_{\xi}$, and $p_{\xi} \leq p$ for $\xi<\kappa^{+}$such that

(1.12) $\beta_{\xi}<\gamma_{\xi}<\beta_{\xi^{\prime}}<\kappa^{+}, \tau_{\xi}<\kappa$ for $\xi<\xi^{\prime}<\kappa^{+}$;

(1.13) $p_{\xi}$ forces that if $f$ is a nonextendable $\tau_{\xi}$-colored embedding into $\left[\gamma_{\xi}, \kappa^{+}\right)$, then there is an edge between $\left[\beta_{\xi}, \gamma_{\xi}\right)$ and $\operatorname{Rng}(f)$.

Again, no problem with the construction arises. By the properties of $P$ we can assume that the $p_{\xi}$ 's are pairwise compatible, $\left\{\operatorname{Dom}\left(p_{\xi}\right): \xi<\kappa^{+}\right\}$form a $\Delta$-system, and that $\tau_{\xi}=\tau$ (by shrinking, if necessary). Put $\gamma=\sup \left\{\gamma_{\xi}: \xi<\kappa^{2}\right\}\left(\kappa^{2}\right.$ again denotes ordinal square). Let $p^{\prime}$ be the restriction of any of the $p_{\xi}$ 's to the kernel of the $\Delta$-system. Obviously, $p^{\prime} \leq p$. Choose a $p^{\prime \prime} \leq p^{\prime}$ with $p^{\prime \prime} \Vdash$ " $f: \alpha \rightarrow\left[\gamma, \kappa^{+}\right)$is a nonextendable, $\tau$-colored embedding" We can even assume (by $<\kappa$-closure of $P$ ) that $p$ fixes also $\alpha, f$. Therefore, $f$ is a real embedding of $X$.

As $\left|\operatorname{Dom}\left(p^{\prime \prime}\right)\right|<\kappa$, the set $\left\{\xi<\kappa^{2}: p_{\xi}\right.$ and $p^{\prime \prime}$ are compatible $\}$ has order-type $\kappa^{2}$. If $p_{\xi}$ and $p^{\prime \prime}$ are compatible, then a common extension forces that there is an edge between $\left[\beta_{\xi}, \gamma_{\xi}\right)$ and $\operatorname{Rng}(f)$. By absoluteness, there must be such an edge in $V$. But then we get, as in the proof of Theorem 1, that $\kappa^{2}$ is the union of $<\kappa$ sets, each of order-type $\leq \kappa$, a contradiction.

To finish the proof of Theorem 2, let $\beta$ be as in the Claim. Define by recursion on $\xi<\kappa, \alpha(\xi), \beta(\xi), \gamma(\xi), p(\xi), \mu(\xi), f_{\xi}$ such that

(1.14) $\beta(\xi)<\gamma(\xi)<\beta\left(\xi^{\prime}\right)<\kappa^{+}, \beta(0)=\beta$, for $\xi<\xi^{\prime}<\kappa$;

(1.15) $f_{\xi}: \alpha(\xi) \rightarrow[\beta(\xi), \gamma(\xi))$ is an embedding;

(1.16) no edge goes across $[\beta, \beta(\xi))$ and $\operatorname{Rng}\left(f_{\xi}\right)$;

(1.17) $p(\xi) \Vdash$ " $f_{\xi}$ is nonextendable, $\mu(\xi)$-colored";

(1.18) for every $q \leq p$ with $\operatorname{Dom}(q) \subseteq \bigcup\{\operatorname{Dom}(p(\varsigma)): \varsigma<\xi\}$ and $\mu<\kappa$ there exists a $\tau>\xi$ with $p(\tau) \leq q, \mu(\tau)=\mu$.

To ensure (1.14-17) the Claim suffices, (1.18) can be met, as $\kappa^{\kappa}=\kappa$. By (1.4), there is a point $\delta$ appropriate for the embeddings $\left\{f_{\xi}: \xi<\kappa\right\}$. Assume that $r \leq p$ and $r \Vdash g(\delta)=\mu$. There is a $\xi<\kappa$ such that if $S=\bigcup\{\operatorname{Dom}(p(\varsigma)): \varsigma<\kappa\}$, then $\operatorname{Dom}(r) \cap S=\operatorname{Dom}(r) \cap(\bigcup\{\operatorname{Dom}(p(\varsigma)): \varsigma<\xi\})$ so, by (1.18), there is a $\xi^{\prime}$ with

(1.19) $p\left(\xi^{\prime}\right)|S \leq r| S$ and $p\left(\xi^{\prime}\right) \Vdash$ " $f_{\xi^{\prime}}$ is a nonextendable $\mu$-colored embedding.".

But by (1.19), $p\left(\xi^{\prime}\right)$ and $r$ are compatible and the common extension forces that $f_{\xi^{\prime}}\left(\alpha\left(\xi^{\prime}\right)\right)=\delta$ is a good extension, a contradiction.

The most natural way of deducing Theorem 2 would be to show that if $|X|=\kappa$, $|Y|=\kappa^{+}$and $Y \hookrightarrow(X)_{\kappa}^{1}$ holds then this remains true if $2^{\kappa}$ is blown up by adding Cohen subsets of $\kappa$. We can prove this only if $X$ is small.

THEOREM 3. Assume that $\kappa^{\kappa}=\kappa,|Y| \leq 2^{\kappa},|X|<\kappa, Y \rightarrow(X)_{\kappa}^{1}$. If $2^{\kappa}$ is enlarged by adding (any number of Cohen subsets of $\kappa$, then $Y \rightarrow(X)_{\kappa}^{1}$ remains valid. There is a graph $Y$ with $|Y|=\left(2^{\kappa}\right)^{+}, \operatorname{Chr}(Y)>\kappa$ but $\operatorname{Chr}(Y) \leq \kappa$ after adding $\left(2^{\kappa}\right)^{+}$Cohen subsets of $\kappa$. 
ProOF. For the example, take the edge graph. The claim will be clear from the following well-known observation (see [9]): the edge graph on $\lambda$ has chromatic number $\leq \kappa$ if and only if $\lambda \leq 2^{\kappa}$.

Now assume that $|Y| \leq 2^{\kappa}$, and forcing by $P$ an $f: 2^{\kappa} \rightarrow \kappa$ with no monocolored $X$ is born. As $P$ is $\kappa^{+}$-c.c., $f$ is already contained in a submodel built from a $P^{\prime} \subseteq P$ with $\left|P^{\prime}\right| \leq 2^{\kappa}$, i.e. we can assume that at most $2^{\kappa}$ Cohen sets are added. Choose for every $\alpha<2^{\kappa}$ a condition $p_{\alpha}$, and a color $i_{\alpha}<\kappa$ with $p_{\alpha} \Vdash f(\alpha)=i_{\alpha}$. For a subset $S \subseteq 2^{\kappa} Y \mid S \longmapsto(X)_{\kappa}^{1}$ holds, and $i_{\alpha}=i$ for $\alpha \in S$.

By a well-known observation $P$ is the union of $\kappa$ centered subsets, so we can even assume that for $\alpha, \beta \in S, p_{\alpha}$ and $p_{\beta}$ are compatible. If $A \subseteq S$ spans a copy of $X$, $p \in P$ extends $\left\{p_{\alpha}: \alpha \in A\right\}$, then $p$ forces that $A$ is monocolored, a contradiction.

THEOREM 4. There exists a graph $Y$ on $2^{\omega}$ with $\operatorname{Chr}(Y) \geq \omega_{1}$ such that $Y$ can be made $\omega$-chromatic with a ccc forcing.

PROOF. Construct $Y$ on $2^{\omega}$ such that for every $A \in\left[2^{\omega}\right]^{\omega}$ (i.e. the order-type of $A$ is $\omega$ ) there is an $\alpha>\sup A, Y(\alpha)=A$, and $\operatorname{tp}(Y(\alpha)) \leq \omega$ for all $\alpha<2^{\omega}$. If $f: 2^{\omega} \rightarrow \omega$, put $N=\left\{i<\omega: f^{-1}(i)\right.$ is bounded in $\left.2^{\omega}\right\}$. For $i \in N$, choose $\gamma_{i}<2^{\omega}$ with $f^{-1}(i) \subseteq \gamma_{i}$. Choose $x_{0}<x_{1}<\cdots$ in such a way that $x_{0}>\sup \left\{\gamma_{i}: i \in N\right\}$, and for every $i \in \omega-N$ there is a $t$ with $f\left(x_{t}\right)=i$ (possible by the definition of $N)$. Now, if $\alpha>\sup \left\{x_{t}: t<\omega\right\}$ has $Y(\alpha)=\left\{x_{0}, x_{1}, \ldots\right\}$ then $f(\alpha) \notin N$ as $\alpha>\gamma_{i}$ $(i \in N)$, so there is a $t<\omega$ with $f\left(x_{t}\right)=f(\alpha)$, i.e. $f$ is not a good coloring.

To make $Y \omega$-chromatic put $P=\left\{p: \operatorname{Dom}(p) \in\left[2^{\omega}\right]^{<\omega}, \operatorname{Rng}(p) \subseteq \omega, p\right.$ good colors $\}, p \leq q$ whenever $p \supseteq q$. If $\left\{p_{\alpha}: \alpha<\omega_{1}\right\}$ are incompatible, we can assume that $\operatorname{Dom}\left(p_{\alpha}\right) \cap \operatorname{Dom}\left(p_{\beta}\right)=s, p_{\alpha}\left|s=p_{\beta}\right| s$, i.e. $\operatorname{Dom}\left(p_{\alpha}\right)-s$ and $\operatorname{Dom}\left(p_{\beta}\right)-s$ are joined. Without loss of generality, all $\operatorname{Dom}\left(p_{\alpha}\right)-s$ are $n$-element, enumerated as $\operatorname{Dom}\left(p_{\alpha}\right)-s=\left\{x_{\alpha}^{0}, x_{\alpha}^{1}, \ldots, x_{\alpha}^{n-1}\right\}$. We can assume that for $\alpha<\beta x_{\alpha}^{i}<x_{\beta}^{i}$ and put $F_{i, j}(\alpha)=\left\{\beta<\omega_{1}: x_{\beta}^{i}<x_{\alpha}^{j}\right.$ and are joined $\}$. Obviously $\operatorname{tp}\left(F_{i j}(\alpha)\right) \leq \omega$ (though not necessarily $\subseteq \alpha$ ), so there is a set $Z \in\left[\omega_{1}\right]^{\omega_{1}}$ independent for every $F_{i j}$, i.e. $\operatorname{Dom}\left(p_{\alpha}\right)-s$ and $\operatorname{Dom}\left(p_{\beta}\right)-s$ are not joined by an edge for $\alpha, \beta \in Z, \alpha \neq \beta$.

Note that the graph $Y$ constructed in the proof was first used by Galvin and Hajnal in an unpublished paper (see also [3, pp. 162-163]).

For the coloring number instead of the chromatic number there are no such examples. As usual, $\operatorname{Col}(Y) \leq \omega$ means that there is a wellordering of the vertex set of $Y$ such that every vertex is joined to only finitely many smaller vertices.

THEOREM 5. Let $Y$ be a graph with $\operatorname{Col}(Y)>\omega$. Then $\operatorname{Col}(Y)>\omega$ holds after forcing by a proper partial ordering.

ProOF. Put $\kappa=|Y|$. We can assume that for every $Y^{\prime} \subseteq Y$ with $\left|Y^{\prime}\right|<\kappa$ $\operatorname{Col}\left(Y^{\prime}\right) \leq \omega$ holds. This implies (by Shelah's theorem) that $\kappa=\operatorname{cf}(\kappa)>\omega$ and that $S=\{\alpha<\kappa: \exists \delta=\delta(\alpha) \geq \alpha$ with $|Y(\delta) \cap \alpha| \geq \omega\}$ is stationary (see [16]).

Assume that $P$ is a proper notion of forcing (see [23]) and $p \Vdash$ " $f: \kappa \rightarrow[\kappa]^{<\omega}$, if $\{\alpha, \beta\} \in Y$, then $\alpha \in f(\beta)$ or $\beta \in f(\alpha)$ " (see [16]). As $P$ is proper, there are finitary functions $P_{n}:[H(\lambda)]^{n} \rightarrow H(\lambda)\left(\lambda=\left(2^{2^{\kappa}}\right)^{+}\right)(n<\omega)$ such that if $N \subseteq H(\lambda),|N|=\omega$ and $N$ is closed under the $F_{n}$ 's then there is a $(P, N)$-generic condition.

There are ordinal functions $G_{n}:[\kappa]^{n} \rightarrow \kappa$ such that if $M \subseteq \kappa,|M|=\omega$ and $M$ is closed under the $G$ 's then there is an $N$, closed under the $F$ 's with $N \cap \kappa=M$. 
As the set $C=\left\{\alpha<\kappa: G_{n}^{\prime \prime}[\alpha]^{n} \subseteq \alpha\right\}$ is closed, unbounded, there is an $\alpha \in C \cap S$. Choose $\delta \geq \alpha$ as in the definition of $S$, and $\left\{\alpha_{0}, \alpha_{1}, \ldots\right\} \subseteq Y(\delta) \cap \alpha$. By the properties of $F, G$ and $C$, there is an $N$, closed under the $F_{n}$ 's, $N \supseteq\left\{\alpha_{0}, \alpha_{1}, \ldots\right\}$, $N \cap \kappa \subseteq \alpha$.

Now choose $q \leq p$ which is $(P, N)$-generic. For $n<\omega, q \Vdash$ "if $\beta \in f\left(\alpha_{n}\right)$, then $\beta \in N$ ". If $q^{\prime} \leq q$ forces for an $n$ that " $\alpha_{n} \notin f(\delta)$ " (as $f(\delta)$ is finite), then $q^{\prime} \Vdash$ " $\delta \in N$ " which is absurd.

Let us note that using the ideas of Theorem 5 and of [2] one can show e.g. that if $\varphi$ is an order-type, $P$ is proper and $V \vDash \varphi \rightarrow(\omega)_{\omega}^{1}$ then $V^{P} \vDash \varphi \rightarrow(\omega)_{\omega}^{1}$, two special cases (ccc and $\omega_{1}$-closed) have been proved and used in [1 and 2].

Under the axiom of constructibility we can get incompactness results, generalizing the main theorem of $[\mathbf{1 5}]$.

THEOREM 6. ( $V=L)$ If $|X| \leq \mu=\operatorname{cf}(\mu), X$ has at least one edge, $\kappa>\mu \geq \omega$ are regular, $\kappa$ is not weakly compact, then there exists a graph $Y$ on $\kappa$, such that

(1.20) $Y \nrightarrow(X)_{\mu}^{1}, Y^{\prime} \nrightarrow(X)_{\mu}^{1}$ for $Y^{\prime} \leq Y,\left|Y^{\prime}\right|<\kappa$;

(1.21) if $\xi<\kappa$ then either $|Y(\xi)|<\mu$ or is of type $\mu$ and is cofinal in $\xi$;

(1.22) if $K_{\alpha} \npreceq X$ then $K_{\alpha} \nless Y$, either.

PROOF. By results of Jensen [5], there exists a stationary set $E \subset\{\xi<$ $\kappa: \operatorname{cf}(\xi)=\mu\}$ with $E \cap \xi$ nonstationary in $\xi$ for every $\xi<\kappa$. We use the following form of the diamond principle for $E$ :

(1.23) there is a sequence $\left\langle S_{\alpha}: \alpha \in E\right\rangle$ such that if $S=\left\langle f_{\beta}: \beta<\kappa\right\rangle$ is such that for $\beta<\kappa, f_{\beta}: \alpha_{\beta} \rightarrow \kappa$ is an increasing function, $\alpha_{\beta}<\mu, \sup \operatorname{Rng}\left(f_{\beta}\right)<$ $\inf \operatorname{Rng}\left(f_{\beta^{\prime}}\right)$, for $\beta<\beta^{\prime}<\kappa$, then for stationary many $\alpha \in E, S_{\alpha}=S \mid \alpha$ holds.

We are going to define $Y(\beta)$ for $\beta<\kappa$ and simultaneously the function $\Phi$ as in Theorem 1. $Y(\beta)=\varnothing$ unless $\beta=\mu \beta \in E, S_{\beta}$ is a sequence of the form $\left\langle f_{\gamma}: \gamma<\beta\right\rangle$ with $f_{\gamma}: \alpha_{\gamma} \rightarrow \beta$ an embedding of $X$ (this is meaningful as $Y, \Phi$ are to be defined on $\beta), A_{\gamma}=\operatorname{Rng}\left(f_{\gamma}\right), \sup A_{\gamma}<\inf A_{\gamma^{\prime}}$ whenever $\gamma<\gamma^{\prime}<\beta$ and no edge goes between two $A_{\gamma}$ 's. In this case, choose a sequence $\left\langle\tau_{\xi}: \xi<\mu\right\rangle$ converging to $\beta$, and take

$$
Y(\beta)=\bigcup\left\{B\left(\mu \tau_{\xi}+\xi\right): \xi<\mu\right\}
$$

where $B(\gamma)=f_{\gamma}^{\prime \prime}\left(\alpha_{\gamma}\right)(\gamma<\beta)$, and, if for $\xi<\mu \varnothing \neq s \subseteq X\left(\alpha_{\mu \tau_{\xi}+\xi}\right)$ spans a complete graph, then take

$$
\Phi\left(f_{\mu \tau_{\xi}+\xi}^{\prime \prime} \cup \cup\{\beta\}\right)=s \cup\left\{\alpha_{\mu \tau_{\xi}+\xi}\right\} .
$$

Obviously, (1.21) holds. This gives the claim exactly as in Theorem 1, which in turn implies the existence of nonextendable monocolored embeddings $f_{\gamma}: \alpha_{\gamma} \rightarrow \kappa$, where $f_{\mu \delta+\xi}$ is $\xi$-colored if $\xi<\mu$, and $\sup \operatorname{Rng}\left(f_{\gamma}\right)<\inf \operatorname{Rng}\left(f_{\gamma^{\prime}}\right)\left(\gamma<\gamma^{\prime}<\kappa\right)$. By (1.23) there is a $\beta=\mu \beta \in E$ with $S_{\beta}=\left\langle f_{\gamma}: \gamma<\beta\right\rangle$. But then (1.24) gives that $\beta$ can get no color. The relation $Y^{\prime} \nrightarrow(X)_{\mu}^{1}$ for $Y^{\prime} \subseteq Y,\left|Y^{\prime}\right|<\kappa$ follows from $\operatorname{Chr}\left(Y^{\prime}\right) \leq \mu$, and this is an easy corollary of (1.21) and the nonstationarity of $E \cap \beta$ for $\beta<\kappa$.

If $\alpha=\omega$, i.e. if the excluded subgraph is $K_{\omega}$ in the results above, a slightly different approach is possible. In this case we can define an ordinal-valued rank function on those finite subsets spanning complete subgraphs: $r_{X}(s)=0$ if no proper end extension of $s$ spans a complete subgraph, otherwise

$$
r_{X}(s)=\sup \left\{r_{X}(s \cup\{\xi\})+1: \sup (s)<\xi, s \cup\{\xi\} \text { spans a complete graph }\right\} .
$$


Using this notion, Theorem 1 will change to the following.

THEOREM 1'. If $K_{\omega} \nless X, X$ is a graph on $\kappa=\operatorname{cf}(\kappa) \geq \omega$, then there exists a graph $Y$ on $2^{\kappa}$ with $K_{\omega} \nless Y, Y \rightarrow(X)_{\kappa}^{1}$, and, moreover $r_{Y}(\varnothing)=r_{X}(\varnothing)+1$ if $r_{X}(\varnothing)$ is limit, $r_{Y}(\varnothing)=r_{X}(\varnothing)$ otherwise.

PROOF. Exactly as in Theorem 1. From that construction it is obvious that if $s \in\left[2^{\kappa}\right]^{<\omega},|s| \geq 2$ spans a complete subgraph in $Y$, then $r_{Y}(s) \leq r_{X}(\Phi(s))$. If $r_{X}(\varnothing)=\beta+1$, then $r_{X}(\{\xi\}) \leq \beta$ for $\xi<\kappa, r_{X}\left(\left\{\xi, \xi^{\prime}\right\}\right)<\beta$ for $\xi \in X\left(\xi^{\prime}\right)$. This gives $r_{Y}\left(\left\{\xi, \xi^{\prime}\right\}\right)<\beta$ for $\xi \in Y\left(\xi^{\prime}\right)$. This implies $r_{Y}(\{\xi\}) \leq \beta$ for $\xi<2^{\kappa}$, i.e. $r_{Y}(\varnothing) \leq \beta+1$. If $r_{X}(\varnothing)$ is limit we only get $r_{Y}(\varnothing) \leq r_{X}(\varnothing)+1$. It is easy to see that if $r_{X}(\varnothing)$ is limit, a $Y$ as in the Theorem must satisfy $r_{Y}(\varnothing) \geq r_{X}(\varnothing)+1$ as othewise $g(\xi)=r_{Y}(\{\xi\})$ would be a bad coloring.

Notice that Theorems 2 and 6 have similar counterparts.

We also mention the following observation.

THEOREM 7. If $\kappa^{\kappa}=\kappa$, then for every $\alpha<\kappa^{+}$there exists a universal graph among those of size $\leq \kappa$ and of rank $\leq \alpha$. There is a $Y$ with $K_{\omega} \$ Y,|Y|=\kappa^{+}$ which embeds every $|X| \leq \kappa$ for which $K_{\omega} \nless X$.

PROOF (IN OUTLINE). One can build a tree-like construction, as in the proof of Theorem 1, replacing $\Phi$ by an ordinal valued "rank function" which associates an ordinal to every finite subset spanning a complete subgraph an ordinal number.

Notice that, as a well-known folklore observation shows, in no $\kappa$ does there exist a universal $\kappa$-sized graph omitting $K_{\omega}$.

2. Obligatory subgraphs. In this section we prove a few results saying that a certain graph $Y$ with $Y \rightarrow(X)_{\omega}^{1}$ or $Y \rightarrow(X)_{\omega}^{2}$ must contain something larger than $X$. Notice that if $Y \rightarrow(X)_{\omega}^{1}$ or $Y \rightarrow(X)_{\omega}^{2}$ implies $Z \leq Y$ then $Y \rightarrow(X)_{\omega}^{1}$ or $Y \rightarrow(X)_{\omega}^{2}$ also implies $Y \rightarrow(Z)_{\omega}^{1}, Y \rightarrow(Z)_{\omega}^{2}$, respectively.

DEFInITION. If $X=\langle V(X), E(X)\rangle$ is a finite graph, $V(X)$ is partitioned as $V(X)=A \cup B \cup C \cup D \cup\{x, y\}, 1 \leq n<\omega$, then $H_{n}(A, B, C, D, x, y)$ is the following graph: for every pair $\langle i, j\rangle$ with $1 \leq i \leq n, 1 \leq j \leq n$ take a disjoint copy of $X, X_{i j}$ on $V\left(X_{i j}\right)=A_{i j} \cup B_{i j} \cup C_{i j} \cup D_{i j} \cup\left\{x_{i j}, y_{i j}\right\}$ then do the following amalgamations: $A_{i_{1} j_{1}}=A_{i_{2} j_{2}}\left(1 \leq i_{1}, i_{2}, j_{1}, j_{2} \leq n\right), C_{i j_{1}}=C_{i j_{2}}\left(1 \leq j_{1}, j_{2} \leq n\right)$, $D_{i_{1} j}=D_{i_{2} j}\left(1 \leq i_{1}, i_{2} \leq n\right)$, and similarly $x_{i j_{1}}=x_{i j_{2}}, y_{i_{1} j}=y_{i_{2} j}$.

We get, therefore, a graph on

$$
\begin{aligned}
& A \cup \bigcup\left\{B_{i j}: 1 \leq i, j \leq n\right\} \cup \bigcup\left\{C_{i}: 1 \leq i \leq n\right\} \cup \bigcup\left\{D_{j}: 1 \leq j \leq n\right\} \\
& \cup \bigcup\left\{x_{i}, y_{j}: 1 \leq i, j \leq n\right\} .
\end{aligned}
$$

THEOREM 8. If $3 \leq|V(X)|<\omega, Y \rightarrow(X)_{\omega}^{1}$, then there exists a decomposition $V(X)=A \cup B \cup C \cup D \cup\{x, y\}$ with $B \neq \varnothing$ such that for every $n<\omega$, $H_{n}(A, B, C, D, x, y) \leq Y$.

PROOF. As $X$ is finite, it is sufficient to show that for every $n<\omega$ there exists an appropriate decomposition. We will use the following easy observation: for every $k, l<\omega$ there is an $N<\omega$ such that if $F_{i j}$ is a $k$-element set for $i, j<N$, then there are $U, V \in[N]^{l}$ such that for $i \in U, j \in V, F_{i j}=A \cup B_{i j} \cup C_{i} \cup D_{j}$ holds 
where all the sets $\left\{A, B_{i j}, C_{i}, D_{j}: i \in U, j \in V\right\}$ are disjoint. This can be proved by a standard $\Delta$-system argument; the proof is left to the reader.

Let $m=m(n)$ be a sufficiently large finite number (we will not calculate it, but there is an effective bound on it). We define a graph $W$ on $V(Y)$ with $\{u, v\} \in E(W)$ if there are $m$ different copies of $X$ in $Y$ containing both $u$ and $v$. If $\operatorname{Chr}(W)>$ $\omega$, then by an old Erdös-Hajnal theorem [7] there are arbitrarily large complete bipartite subgraphs of $W$. If $m$ is large enough, there are $2 N$ vertices, $\left\{x_{i}, y_{i}\right.$ : $1 \leq i \leq N\}$ such that $\left\{x_{i}, y_{j}\right\}$ is covered by a copy of $X, X_{i j}$ with $x_{i}, y_{j}$ playing the roles of the same pair of vertices, and every $V\left(X_{i j}\right)$ contains a vertex not covered by $\bigcup\left\{X_{s t}: s, t \neq i, j\right\}$. Applying the result mentioned at the beginning of the proof we get the result; one only must notice that $B \neq \varnothing$ by the noncovering property of the $V\left(X_{i j}\right)$ sets.

If $\operatorname{Chr}(W) \leq \omega, V(Y)$ can be covered by countably many $W$-independent sets; one of them must arrow $X$. We can, therefore, assume that $V(Y)$ is $W$-independent, i.e. no pair is contained in $m$ copies of $X$. Again, by considering those pairs which are covered by at least one copy of $X$, we get that there are vertices $\left\{x_{i}, y_{i}: 1 \leq\right.$ $i \leq N\}$ with $x_{i}, y_{j}$ covered by $X_{i j}$ with $V\left(X_{i j}\right)=A \cup B_{i j} \cup C_{i} \cup D_{j} \cup\left\{x_{i}, y_{j}\right\}$. Now, if $A \cup \bigcup\left\{C_{i}: 1 \leq i \leq N\right\} \cup \bigcup\left\{D_{i}: 1 \leq i \leq N\right\} \neq \varnothing$, say $a \in A \cup C_{i}$, then $\left\{a, x_{i}\right\}$ is covered by $X_{i j}(1 \leq j \leq N)$, a contradiction, if $N \geq m$. We get, therefore, that $V\left(X_{i j}\right)=B_{i j} \cup\left\{x_{i}, y_{j}\right\}$, i.e. a decomposition with $A=C_{i}=D_{i}=\varnothing$.

The simplest instance of interest of Theorem 8 is when $X$ is the triangle, i.e. $K_{3}$; then $H_{n}$ is the graph on $\left\{x_{i}, y_{j}, z_{i j}: 1 \leq i, j \leq n\right\}$ with edges of the form $\left\{x_{i}, y_{j}\right\}$, $\left\{x_{i}, z_{i j}\right\},\left\{z_{i j}, y_{j}\right\}$. One is tempted to think that this can be strengthened and $K_{4}^{-}$, the complete four-graph minus an edge, is also obligatory. Though this is true for $|Y|=\omega_{1}[7]$ there is a counterexample of size $2^{2^{\omega}}$, as is shown in [6]. We show that this phenomenon can appear at larger cardinalities. We need some preliminary (finite) constructions.

LEMMA 1. Assume that $k \geq 3,0<\theta<\frac{1}{2}, x$ is a large enough natural number. Then there exists a sequence of disjoint sets, $T_{0}, T_{1}, \ldots$ and a $k$-hypergraph $\mathscr{H}$ such that

(2.1) $\left|T_{0}\right|=x,\left|T_{1}\right| \geq[x /(k-1)],\left|T_{i+1}\right| \geq\left|T_{i}\right|^{1+\theta}(i \geq 1)$;

(2.2) for every $H \in \mathscr{H}$ there is an $i \geq 1$ such that $\left|H \cap T_{i}\right|=1,\left|H \cap T_{i-1}\right|=k-1$, for every $p \in T_{i}$ there is an $H \in \mathscr{H}$ with $p \in H, H-\{p\} \subseteq T_{i-1}(i \geq 1)$;

(2.3) $\mathscr{H}$ is $\leq 3$-circuitless;

(2.4) $\left\{H \cap T_{0}: H \in \mathscr{H}\right\}$ are disjoint.

Proof. To start, choose $T_{0}, T_{1}$ with $\left|T_{0}\right|=x,\left|T_{1}\right|=[x /(k-1)]$, and build $\mathscr{H}$ on $T_{0} \cup T_{1}$ as follows: for $p \in T_{1}$ choose $f(p) \in\left[T_{0}\right]^{k-1}$ with $f(p) \cap f(q)=\varnothing$ whenever $p \neq q$, and put $\left\{\{p\} \cup f(p): p \in T_{1}\right\}$ into $\mathscr{H}$. We continue by building $T_{2}, T_{3}, \ldots$ and $f(p) \in\left[T_{i-1}\right]^{k-1}$ for $p \in T_{i}(i \geq 2)$ with the following extra condition:

(2.5) $T_{i}=\bigcup\left\{T_{i, j}: 1 \leq j \leq k-1\right\},\left|T_{i j}\right|=\left|T_{i}\right| /(k-1)$, and, if $p \in T_{i j_{0}}, q \in T_{i j_{1}}$, $j_{0} \neq j_{1}$ then $f(p) \cap f(q)=\varnothing$.

Assume that the hypergraph on $T_{0} \cup \cdots \cup T_{i}$ has already been constructed. First, partition each $T_{i j}(1 \leq j \leq k-1)$ into $k-1$ equal parts, $T_{i j}=\bigcup\{T(i, j, l): 1 \leq$ $l \leq k-1\}$. For every $l$ with $1 \leq l \leq k-1$ we shall construct a $k-1$-hypergraph $\mathscr{H}_{l}$ on $\bigcup\{T(i, j, l): 1 \leq j \leq k-1\}$ such that if $H \in \mathscr{H}_{l}$, then

$$
|H \cap T(i, j, l)|=1 \quad(1 \leq j \leq k-1),
$$


and $\mathscr{H}_{l}$ is $\leq 3$-circuitless. Next, we choose for every $H \in \mathscr{H}_{l}$ a new vertex $p \in$ $T(i+1, l)$ for which $f(p)=H$ will hold. By an observation of Erdös-Hajnal [7] such an $\mathscr{H}_{l}$ exists with $\left|\mathscr{H}_{l}\right|>\left|T_{i}\right|^{1+\theta}$ provided that $0<\theta<\frac{1}{2}$ and $\left|T_{i}\right|$ is large enough. We shall, therefore, have $\left.\left|T_{i+1}=\sum\right| \mathscr{H}_{l}|>| T_{i}\right|^{1+\Theta}$.

We shall have to prove that the hypergraph is $\leq 3$-circuitless. Clearly, two edges can have at most one vertex in common. Assume that $H_{1}, H_{2}, H_{3}$ form a 3-circuit, $H_{r}=f\left(p_{r}\right) \cup\left\{p_{r}\right\}(r=1,2,3)$. If $p_{1}, p_{2}, p_{3} \in T_{i}(i \geq 1)$ we are done, as by assumption, $f\left(p_{1}\right), f\left(p_{2}\right), f\left(p_{3}\right)$ cannot form a 3-circuit. If $p_{1}, p_{2} \in T_{i}, p_{3} \in T_{i-1}$, then clearly $\left|H_{3} \cap\left(H_{1} \cup H_{2}\right)\right|=1$ (contains only $p_{3}$ ), so $H_{1}, H_{2}, H_{3}$ is not a 3 circuit. If $p_{1} \in T_{i}, p_{2}, p_{3} \in T_{i-1}$, then the only possibility is that $p_{2}, p_{3} \in f\left(p_{1}\right)$, and $f\left(p_{2}\right) \cap f\left(p_{3}\right) \neq \varnothing$, i.e. $p_{2} \in T_{i-1, j_{0}}, p_{3} \in T_{i-1, j_{1}}$ with $j_{0}=j_{1}$ and $j_{0} \neq j_{1}$ at the same time, an impossibility.

LEMMA 2. For $k \geq 3$ there exists a finite, 3 -circuitless $k$-hypergraph $\mathscr{H}$ on $S=\bigcup \mathscr{H}$ with the following property: if $X \subset S, X \neq \varnothing$ then there exists an $H \in \mathscr{H}$ such that either $H \subseteq X$ or $|H \cap X|=1$ holds.

PROOF. First choose a $y<\omega$ so large that there exists a 3 -chromatic $\leq 3$ circuitless $k-1$-hypergraph of $y$ edges (possible by Erdös-Hajnal [7]). Choose $x$ so large that $x^{\theta}>y$, where $0<\theta<\frac{1}{2}$ fixed, and $x$ satisfies Lemma 1 . Construct a sequence of disjoint sets $T_{0}, T_{1}, \ldots, T_{r}$ with $\left|T_{0}\right| \geq x, \ldots,\left|T_{r}\right| \geq k \cdot x^{y}$ and build the $\leq 3$-circuitless system of Lemma 1 on $T_{0} \cup T_{1} \cup \cdots \cup T_{r}$. By a probabilistic construction (see $[\mathbf{2 1}]$ ) we can add edges in $\left[T_{0}\right]^{k}$ extending it to a system where every vertex of $T_{0}$ is contained in at least $x^{\theta} \geq y$ hyperedges. Choose a set $S$ with $S \cap\left(T_{0} \cup \cdots \cup T_{r}\right)=\varnothing$ and $|S|=y\left(\begin{array}{l}x \\ y\end{array}\right)$, and continue extending the hypergraph by hyperedges of type $\{s\} \cup A_{s}$ with $A_{s} \in\left[T_{r}\right]^{k-1}, A_{s} \cap A_{s^{\prime}}=\varnothing$ for $s \neq s^{\prime}\left(s, s^{\prime} \in S\right)$.

Call a set $A$ independent if $|H \cap A| \leq 1$ holds for every hyperedge $H$ constructed so far. For every $y$-element independent subset $A \subseteq T_{0}$ choose a subset $R_{A} \subseteq S$, $\left|R_{A}\right|=y$ in such a way that $R_{A} \cap R_{A^{\prime}}=\varnothing$ if $A \neq A^{\prime}$. Build a 3-chromatic $\leq 3$ circuitless $k-1$-hypergraph $\mathscr{K}$ on $R_{A}$ and choose a vertex $t(K) \in A$ for every $K \in \mathscr{K}$, again with $t(K) \neq t\left(K^{\prime}\right)$ for $K \neq K^{\prime}$.

A straightforward checking shows that the hypergraph finally constructed is $\leq 3$ circuitless. Assume that $X \neq \varnothing$ is a subset of $\bigcup T_{i} \cup S$ such that if $H$ is a hyperedge, then neither $H \subseteq X$ nor $|H \cap X|=1$ holds. If $T_{0} \cap X=\varnothing$ then we get by induction that $T_{i} \cap X=\varnothing$ and at last $S \cap X=\varnothing$, i.e. $X=\varnothing$. If $p \in X \cap T_{0}$, then there are $y$ hyperedges $H_{1}, \ldots, H_{y}$ with $p \in H_{i}$, so, as $\left|X \cap H_{i}\right| \neq 1$, there are other vertices $q_{i} \in H_{i} \cap X-\{p\}$. The set $A=\left\{q_{1}, \ldots, q_{y}\right\} \subseteq X \cap T_{0}$ is independent (as our system is $\leq 3$-circuitless), so there is an appropriate $R_{A} \subseteq S$. As the $k-1$-hypergraph on $R_{A}$ is 3-chromatic, either there is a $K \subseteq X$ so $t(K) \cup K \subseteq X$, or there is a $K$ with $K \cap X=\varnothing$ (again, $|X \cap(\{t(K)\} \cup K)|=1)$.

THEOREM 9. There are finite graphs $T_{1}, T_{2}, \ldots$ such that

(2.6) $K_{4}^{-} \nless T_{n}(n=1,2, \ldots)$;

(2.7) if $|Y| \leq \omega_{n}$, and $Y \rightarrow\left(T_{n}\right)_{\omega}^{1}$, then $K_{4}^{-} \leq Y$.

PROOF. For $n=1$ we can take the triangle (see [7]). We are going to construct a graph $T_{n}$ with (2.6-7) and with the additional property that $V\left(T_{n}\right)$ is covered by the triangles contained in $T_{n}$. This certainly holds for $n=1$. Put $k=\left|V\left(T_{n-1}\right)\right|$. By Lemma 2, there exists a $k$-hypergraph $\mathscr{H}$ on a finite set $S$ which is $\leq 3$-circuitless, 
and if $X \subseteq S$ is nonempty, then there exists an $H \in \mathscr{H}$ such that either $H \subseteq X$ or $|H \cap X|=1$ holds. For every $H \in \mathscr{H}$, fix a bijection $f_{H}: V\left(T_{n-1}\right) \rightarrow H$, and take $T_{n}=\bigcup\left\{f_{H}^{\prime \prime} E\left(T_{n-1}\right): H \in \mathscr{H}\right\}$, i.e. draw a copy of $T_{n-1}$ inside every $H$. We claim that $T_{n}$ is appropriate. As $\mathscr{H}$ is $\leq 3$-circuitless, all triangles of $T_{n}$ come from triangles of the copies of $T_{n-1}$, and using that $\mathscr{H}$ is 2 -circuitless, we get that $K_{4}^{-} \nless T_{n}$. It is also obvious that every vertex of $S$ is covered by a triangle.

We show that if $Y$ is a graph on $\omega_{n}, K_{4}^{-} \not Y$, then $Y \nrightarrow\left(T_{n}\right)_{\omega}^{1}$. Decompose $\omega_{n}=V(Y)$ into a continuous, increasing union of sets $\left\{V_{\alpha}: \alpha<\omega_{n}\right\}$ with $\left|V_{\alpha}\right| \leq$ $\omega_{n-1}$ such that, if $x_{1}, x_{2} \in V_{\alpha}$, and there exists a $y$ joined to both $x_{1}$ and $x_{2}$, then there is such a $y$ in $V_{\alpha}$. Color each $V_{\alpha+1}-V_{\alpha}$ by countably many colors, without a monocolored $T_{n-1}$ (possible, as $\left|V_{\alpha+1}-V_{\alpha}\right| \leq \omega_{n-1}$ ). Assume that $Y \mid A$ contains a monocolored copy of $T_{n}$. By the construction of $T_{n}$, there is a bijection $g: S \rightarrow A$ such that a $T_{n-1}$ is drawn into $g^{\prime \prime} H$ for every $H \in \mathscr{H}$. Let $\alpha$ be the minimal $\beta<\omega_{n}$ such that $A \subseteq V_{\beta+1}$. As the coloring of $V_{\alpha+1}-V_{\alpha}$ is $T_{n-1}$-free, for no $H \in \mathscr{H}$, does $g^{\prime \prime} H \subseteq V_{\alpha+1}-V_{\alpha}$ hold. This gives that if $X=\left\{s \in S: g(s) \in V_{\alpha+1}-V_{\alpha}\right\}$, then $X \neq \varnothing$ and $H \nsubseteq X$ for $H \in \mathscr{H}$. By Lemma 2, there is an $H \in \mathscr{H}$ with $|H \cap X|=1$. As the vertex corresponding to the unique element of $H \cap X$ is covered by a triangle, there are vertices $x_{1}, x_{2} \in V_{\alpha}, y \in V_{\alpha+1}-V_{\alpha}$, forming a triangle. By the closure property of $V_{\alpha}$, there is a $z \in V_{\alpha}$ with $\left\{x_{1}, z\right\},\left\{x_{2}, z\right\} \in E(Y)$, i.e. $\left\{x_{1}, x_{2}, y, z\right\}$ forms a $K_{4}^{-}$.

A natural counterpart to Theorem 9 is given by the following theorem of ErdösGalvin-Hajnal [6]: if $T$ is a finite graph, $K_{4}^{-} \nless T,|V(T)|=n$, there is a graph $Y$ on $V(Y)=\exp _{n}(\omega)$ such that $K_{4}^{-} \not Y Y, Y \rightarrow(T)_{\omega}^{1}$.

THEOREM 10. (a) If $T_{1}, T_{2}, \ldots$ are as in Theorem 9 , and $Y$ is a graph with $K_{4}^{-} \nless Y$, then $Y \nrightarrow\left(T_{1}, T_{2}, \ldots\right)^{1}$.

(b) There is a countable graph $T$ with $K_{4}^{-} \nless T$, and, if $K_{4}^{-} \nless Y Y$, then $Y \nrightarrow(T)_{\omega}^{1}$.

PROOF. (b) trivially follows from (a). One can show (a) by transfinite induction on $\kappa=|V(Y)|$. Decompose $V(Y)=\bigcup\left\{V_{\alpha}: \alpha<\kappa\right\}$ as in Theorem 9, then color $V_{\alpha+1}-V_{\alpha}$ by $1,2, \ldots$ with no $i$-colored $T_{i-1}$. Now proceed as in Theorem 9 .

It is possible to give a theorem similar to Theorem 8 for the case of edge-arrowing. We mention one simple instance.

THEOREM 11. If $Y \rightarrow\left(K_{3}\right)_{\omega}^{2}$, then $Y$ contains every finite 3-chromatic graph.

ProOF. We prove by induction on $\kappa=|V(Y)|$ that if $Y$ does not contain the complete tripartite graph on $n, n, n$ vertices, then $Y \nrightarrow\left(K_{3}\right)_{\omega}^{2}$. Decompose $V(Y)=\bigcup\left\{V_{\alpha}: \alpha<\kappa\right\}$ into an increasing, continuous union with $\left|V_{\alpha}\right|<\kappa$, and, if for $A \in\left[V_{\alpha}\right]^{2 n}, A$ contains the complete bipartite graph on $n, n$ vertices, then all vertices joined to every vertex in $A$ are in $V_{\alpha}$ (possible, as their number is at most $n-1)$. By the inductive hypothesis, the edges inside the $V_{\alpha}$ 's can be countably colored without a monocolored triangle. It suffices, therefore, to color the crossing edges. If $x \in V_{\alpha+1}-V_{\alpha}$, and $U=\left\{y \in V_{\alpha}:\{x, y\} \in E(Y)\right\}$, then $Y$ on $U$ does not contain a complete, bipartite graph on $n, n$ vertices; therefore, by the Erdös-Hajnal theorem mentioned above, $U$ spans a countable chromatic graph. This colors the edges between $x$ and $U$, and this gives a coloring without a monocolored triangle.

3. Edge-partitions. In this section we deal with the case when the edge-set of a graph $Y$ (denoted as $E(Y)$ ) is colored and a monocolored induced copy of 
the target graph is to be found. Using a method of Shelah [22], we show that the Ramsey property of this kind is - consistently - false for uncountable target graphs.

THEOREM 12. It is consistent that there exists a bipartite graph on $\omega, \omega_{1}$ vertices, $X$, such that $Y \mapsto(X)_{2}^{2}$ holds for no $Y$.

Proof. Let $V$ be a model of $\mathrm{ZFC}+\mathrm{CH}$. Extend it by the usual Cohen forcing, i.e. by $P=\{p: \operatorname{Dom}(p)<\omega, \operatorname{Rng}(p) \subseteq\{0,1\}\}$. If $G \subseteq P$ is generic, then in $V[G], \mathrm{CH}$ will still hold; therefore there is a scale $\left\langle f_{\alpha}: \alpha<\omega_{1}\right\rangle$ in it, i.e. for every $f: \omega \rightarrow \omega$ there is an $f_{\alpha}$ eventually dominating it. Fix a name for this sequence. Define $X \subseteq \omega \times \omega_{1}$ as follows: $\langle n, \alpha\rangle \in E(X)$ if and only if $G\left(f_{\alpha}(n)\right)=1$ (here $G: \omega \rightarrow\{0,1\}$ denotes the generic function).

Assume that $1 \Vdash$ " $Y$ is a graph on $\lambda$ ". For $\{\alpha, \beta\} \in E(Y)$ let $p \in G$ be the (unique) shortest condition with $p \Vdash\{\alpha, \beta\} \in E(Y)$. Let $n=\operatorname{Dom}(p)$, and put $f(\alpha, \beta)=G(n)$. Assume that $p \Vdash " g: \omega \rightarrow \lambda, h: \omega_{1} \rightarrow \lambda$ embeds $X$ into the $i$ th color of $Y$ ". For $\alpha<\omega_{1}$ choose a $p_{\alpha} \leq p$ deciding a value of $h(\alpha)$ (i.e. $p_{\alpha} \Vdash$ " $h(\alpha)=\xi$ " for some $\xi<\lambda)$. For an $S \in\left[\omega_{1}\right]^{\omega_{1}}, p_{\alpha}=p^{\prime}(\alpha \in S)$. Choose $G \subseteq P$ with $p^{\prime} \in G$. In $V[G], f(n)=\min \left\{i: G \mid i\right.$ decides $\left.g(n), i>\operatorname{length}\left(p^{\prime}\right)\right\}$ defines an $\omega \rightarrow \omega$ function. There exists, therefore, a $p^{\prime \prime} \leq p^{\prime}$ and an $\alpha \in S$ such that $p^{\prime \prime} \Vdash " f_{\alpha}(n)>f(n)$ for $n \geq n_{0}$ ". We know that $G \mid f(n)$ decides the value of both $g(n)$ and $h(\alpha)$. As $q \leq G \mid f(n)$ with $\operatorname{Dom}(q)=f_{\alpha}(n)+1, q\left(f_{\alpha}(n)\right)=1$ is a shortest condition forcing $\langle n, \alpha\rangle \in E(X) . q$ is also a shortest condition forcing $\{g(n), h(\alpha)\} \in E(Y)$. But if $q^{\prime} \leq q$ with $q^{\prime}\left(f_{\alpha}(n)+1\right)=1-i$, then $q^{\prime} \Vdash$ " $f(g(n), h(\alpha))=1-i$ ", a contradiction.

Using this model we show that the natural counterpart to Theorem 1 may also be false. First we need a rather technical lemma.

LEMMA $3(\mathrm{CH})$. Let $P=\left\{p_{i}: i<\omega\right\}$ be the Cohen forcing. There are sets $F\left(p_{i}\right) \subseteq\left[\omega_{1}\right]^{2}$ with

(3.1) $q \leq p$ implies $F(q) \supseteq F(p)$;

(3.2) if $X \in\left[\omega_{1}\right]^{3}, i<\omega$, then $[X]^{2} \nsubseteq F\left(p_{i}\right)$;

(3.3) for every $g: \omega_{1} \rightarrow \omega$ there are $i<\omega, S \in\left[\omega_{1}\right]^{\omega}$ with $[S]^{2} \subseteq \tilde{F}\left(p_{i}\right)$, $S \subseteq g^{-1}(i)$, where $\tilde{F}(p)=\bigcup\{F(q): q \leq p\}$.

PROOF. We give a construction similar to the one in Theorem 1. Assume that $s_{i} \in\left[\omega_{1}\right]^{<\omega}(i<\omega)$ with the following properties.

(3.4) $\sup \left(s_{i}\right)<\min \left(s_{i+1}\right),\left(s_{i} \times s_{j}\right) \cap \tilde{F}(1)=\varnothing(i<j),\left[s_{i}\right]^{2} \subseteq F\left(p_{i}\right)$, then there exists a point $\alpha>\sup \left(\bigcup\left\{s_{i}: i<\omega\right\}\right)$ such that for every $i<\omega$ there are disjoint conditions $p_{i k} \leq p_{i}\left(k<\left|s_{i}\right|\right)$ such that if the $k$ th element of $s_{i}$ is $y$, then $\{y, \alpha\} \in F\left(p_{i k}\right)$, and if $y \notin \bigcup s_{i}$, then $\{y, \alpha\} \notin \tilde{F}(1)$.

Notice that this saves (3.2). We also demand that every $\alpha$ is used up at a step as above; this insures that $\{y<\alpha:\{y, \alpha\} \in \tilde{F}(1)\}$ has order-type $\leq \omega$. Assume that $g$ does not satisfy $(3.3)$.

ClaIM. There is a $\beta<\omega_{1}$ such that for every $\gamma>\beta, i<\omega$, there is an $s \in\left[\omega_{1}-\gamma\right]^{<\omega}, s \subseteq g^{-1}(i),[s]^{2} \subseteq \tilde{F}\left(p_{i}\right),[\beta, \gamma] \times s \cap \tilde{F}(1)=\varnothing$ and

(3.5) for no $t \geq s, t \neq s, \sup (s)<\min (t-s)$, do both $t \subseteq g^{-1}(i),[t]^{2} \subseteq \tilde{F}\left(p_{i}\right)$ hold.

PROOF OF CLAIM. If not, there are $\left\{\beta_{\xi}, \gamma_{\xi}: \xi<\omega_{1}\right\}$ and $i_{\xi}<\omega$ such that if $s$ satisfies (3.5) and $s \subseteq\left[\gamma_{\xi}, \omega_{1}\right)$ then $\left[\beta_{\xi}, \gamma_{\xi}\right) \times s \cap \tilde{F}(1) \neq \varnothing$. Now proceed as in the Claim of Theorem 1. 
Choose $\beta$ as in the Claim, and select $s_{0}, s_{1}, \ldots$ satisfying (3.5), $s_{i} \subseteq g^{-1}(i)$, $\left[s_{i}\right]^{2} \subseteq \tilde{F}\left(p_{i}\right)$, and satisfying (3.4). As there is an $\alpha$ with $s_{i} \times\{\alpha\} \subseteq \tilde{F}\left(p_{i}\right)$, if $g(\alpha)=i, s_{i} \cup\{\alpha\}$ extends $s_{i}$.

THEOREM 13. It is consistent that there exists a triangle-free graph $X$ of size $\omega_{1}$ such that if $Y \rightarrow(X)_{\omega}^{2}$, then $Y$ contains a $K_{\omega}$, and no $Y$ satisfies $Y \hookrightarrow(X)_{\omega}^{2}$.

Proof. Let $V$ be a model of $\mathrm{CH}, P=\left\{p_{i}: i<\omega\right\}$ the Cohen-real forcing, $G$ a generic set. In $V[G]$, define $X$ as $\bigcup\{F(p): p \in G\}$ where $\{F(p): p \in P\}$ is the system of Lemma 3. (3.1) and (3.2) give that $X$ is triangle free.

Assume that $1 \Vdash$ " $Y$ is a graph on $\lambda$ ". For $\{\beta, \alpha\} \in Y$ choose $i<\omega$ as its color, if $p_{i}$ is the shortest $p \in G$ with $p \Vdash$ " $\{\beta, \alpha\} \in Y$ ". Assume that $p \Vdash$ " $f: \omega_{1} \rightarrow \lambda$ embeds $X$ into the $k$ th color". Obviously, $p \leq p_{k}$, as $p \in G$ implies $p_{k} \in G$.

For every $\alpha<\omega_{1}$ choose a $p^{\alpha} \leq p$ with $p^{\alpha} \Vdash$ " $f(\alpha)=\gamma_{\alpha}$ ". Put $g(\alpha)=i$ if $p^{\alpha}=p_{i}$, and apply Lemma 3. We get that there are $i<\omega$ and $\left\{\alpha_{t}: t<\omega\right\}$ with $\left\{\alpha_{t}, \alpha_{s}\right\} \in \tilde{F}\left(p_{i}\right)$ and $g\left(\alpha_{t}\right)=i$. If $t<s<\omega$ and $\left\{\alpha_{t}, \alpha_{s}\right\} \in F(q)$ with $q \leq p_{i} \leq p \leq p_{k}$, then $q \Vdash$ " $\left\{\alpha_{t}, \alpha_{s}\right\} \in E(X)$ ", so $q \Vdash$ " $\left\{\gamma_{\alpha_{t}}, \gamma_{\alpha_{s}}\right\} \in E(Y)$ and is $k$-colored", so $p_{k} \Vdash$ " $\left\{\gamma_{\alpha_{t}}, \gamma_{\alpha_{s}}\right\} \in E(Y)$ ", i.e. $p_{k}$ forces that $\left\{\gamma_{\alpha_{t}}: t<\omega\right\}$ spans a $K_{\omega}$ in $Y$.

The next theorem uses a different kind of approach. Instead of forcing a suitable target graph $X$ we can make almost all sufficiently complicated graphs $X$ satisfy $(0.3)$ at least for $Y$ 's of restricted size.

THEOREM 14. It is consistent that $2^{\omega}=\omega_{1}, 2^{\omega_{1}}=2^{\omega_{2}}=\omega_{3}$, and $Y \nrightarrow(X)_{2}^{2}$ for every $|Y| \leq \omega_{2}$ where $X$ is any bipartite graph on $\omega \times \omega_{1}$ satisfying

(3.6) if $\alpha \neq \beta, K_{\alpha} \Delta K_{\beta}$ is infinite, where for $\alpha<\omega_{1}, K_{\alpha}=\{n<\omega:\langle n, \alpha\rangle \in$ $E(X)\}$.

ProOF. Let $V$ be a model of GCH. We are going to define a countable support iteration $\left\langle P_{\alpha}, Q_{\alpha}: \alpha<\omega_{3}\right\rangle$ with the property that if $X \in V^{P_{\alpha}}$ satisfies (3.6) and $Y \in V^{P_{\alpha}}$ is a graph on $\omega_{2}$, then $Q_{\alpha}$ gives a two-coloring witnessing $Y \nrightarrow(X)_{2}^{2}$. By GCH in $V$, and $\omega_{2}$-c.c. of $P_{\omega_{3}}$ we can treat every appropriate pair $\langle X, Y\rangle$ at some point $\alpha<\omega_{3}$.

Given a graph $Y$ on $\omega_{2}$, let $Q$ be the following partial order: $p \in Q$ if $p=$ $\langle S, f, \mathscr{H}\rangle, S \in\left[\omega_{2}\right]^{\aleph_{0}}, f: E(Y) \cap[S]^{2} \rightarrow\{0,1\}, \mathscr{H}$ is a countable family of pairs of the form $\langle A, B\rangle$ with $A \subseteq B \subseteq S, \operatorname{tp}(B)$ limit, $A$ cofinal in $B$, and

(3.7) there is no $x \in S-B$ for which $A=E(Y) \cap(B \times\{x\})$ and $f^{\prime \prime} A \times\{x\}=\{i\}$ both hold for some $i \in\{0,1\}$.

The ordering on $Q$ is defined by $\left\langle S^{\prime}, f^{\prime}, \mathscr{H}^{\prime}\right\rangle \leq\langle S, f, \mathscr{H}\rangle$ if $S^{\prime} \supseteq S, f^{\prime} \supseteq f$, and $\mathscr{H}^{\prime} \supseteq \mathscr{H}$. It is immediately seen that this is an $\omega_{1}$-closed partial ordering.

Next we show that for $\xi\left\langle\omega_{2}\right.$ the set $D=\{\langle S, f, \mathscr{H}\rangle: \xi \in S\}$ is dense. To get an extension of the type $\left\langle S \cup\{\xi\}, f^{\prime}, \mathscr{H}\right\rangle$ we need to define $f^{\prime}$ on $E(Y) \cap(S \times\{\xi\})$ without an $\langle A, B\rangle \in \mathscr{H}$ such that $f^{\prime \prime \prime} A \times\{\xi\}$ is one-element. As $|A|=\omega$ and there are countably many such $A$ 's we can do this by diagonalization.

Assume that $p \Vdash$ " $W \times Z$ is an $i$-colored induced copy of $X, g: \omega \rightarrow U$ is the embedding" with $g \in V$. As the order-type of $\operatorname{Rng}(g)$ is a countable ordinal, and for every $\alpha<\omega_{1}$, as $K_{\alpha}=\{n<\omega:\langle n, \alpha\rangle \in X\}$, there is a maximal limit point of $g^{\prime \prime} K_{\alpha}$; for $\omega_{1}$ of the $\alpha$ 's this limit point is the same, $\gamma$, say. By condition (3.6) the sets $\left(g^{\prime \prime} K_{\alpha}\right) \cap \gamma$ are different for these $\alpha$ 's. We can, therefore, choose an $\alpha$ with 
$A=\gamma \cap g^{\prime \prime} K_{\alpha}$ different from $T_{x}=\{y \in B:\{x, y\} \in E(Y)\}$ for every $x \in S$ where $B=\gamma \cap \operatorname{Rng}(g)$. Observe that the order-type of $B$ is a limit ordinal, and $A$ is cofinal in $B$. Also, for no $x \in S-B$, does $A=T_{x}$ hold. Therefore, the condition $\langle S, f, \mathscr{H} \cup\{\langle A, B\rangle\}\rangle$ extends $p$ and clearly forces that no point can be the image of the $\alpha$ th vertex of the uncountable part of $X$.

We show that $Q$ has the $\omega_{2}$-c.c. Assume that $\left\langle S_{\xi}, f_{\xi}, \mathscr{H}_{\xi}\right\rangle \in Q\left(\xi<\omega_{2}\right)$. By usual $\Delta$-system arguments we can find two members, say, $p$ and $q$ such that they are (as ordered structures) isomorphic, if $p=\langle S, f, \mathscr{H}\rangle, q=\langle T, g, \mathscr{K}\rangle, \gamma=\sup (S \cap T)$, then $p|\gamma=q| \gamma, \sup (S)<\min (T-\gamma)$. To get a common extension we have to color the edges of $Y$ between $S-\gamma$ and $T-\gamma$. We have to care about $\langle A, B\rangle \in \mathscr{H} \cup \mathscr{K}$ in (3.7). If $\sup (B) \leq \gamma$ no problem arises; (3.7) will surely hold. If $\sup (B)>\gamma$, then $A-\gamma$ is infinite, so by a countable diagonalization we can satisfy (3.7).

We have, therefore, defined the iteration $P_{\omega_{3}}$. It is obviously $\omega_{1}$-closed, so we only need to show that it has the $\omega_{2}$-c.c. Assume that $p_{\xi} \in P_{\omega_{3}}, Y_{\tau}$ is the $Y$ graph of $Q_{\tau}, S(\xi, \tau)$ is the first component of $p_{\xi}(\tau)$. As every $P_{\tau}$ is $\omega_{1}$-closed, we can assume that both $S(\xi, \tau)$ and $E\left(Y_{\tau}\right) \cap[S(\xi, \tau)]^{2}$ are decided by $p_{\xi} \mid \tau$ for every $\tau \in \operatorname{supp}\left(p_{\xi}\right)$. We can also assume that $\left\{\operatorname{supp}\left(p_{\xi}\right): \xi<\omega_{2}\right\}$ form a $\Delta$-system, i.e. $\operatorname{supp}\left(p_{\xi}\right) \cap \operatorname{supp}\left(p_{\varsigma}\right)=T$ for $\xi<\varsigma<\omega_{2}$. We can-by further shrinking, if needed-assume, that for $\tau \in T$ the conditions $p_{\xi}(\tau)$ are isomorphic (by $\mathrm{CH}$ ), and form an ordered $\Delta$-system, as in the previous proof. Now, we can find a common extension of $p_{\xi}, p_{\varsigma}$ by recursion on $\tau \in \operatorname{supp}\left(p_{\xi}\right) \cup \operatorname{supp}\left(p_{\varsigma}\right)$.

To finish the proof we only need to show that once a coloring witnessing $Y$ $(X)_{2}^{2}$ is found it will still witness this in $V^{P_{w_{3}}}$. This follows from the following easy claim: if $|X| \leq \omega_{1}$ and $Y \leftrightharpoons(X)_{2}^{2}$ is witnessed by $f$, then $f$ will still witness this in any forcing extension done by an $\omega_{1}$-closed partial ordering.

ADDED IN PROOF. In recent unpublished work S. Shelah proved that it is consistent that for every $X$, $\gamma$ there exists a $Y$ with $Y \hookrightarrow(X)_{\gamma}^{2}$, assuming the existence of a proper class of measurable cardinals.

\section{BIBLIOGRAPHY}

1. J. E. Baumgartner and A. Hajnal, A proof (involving Martin's axiom) of a partition relation, Fund. Math. 78 (1973), 193-203.

2. J. E. Baumgartner, A new class of order types, Ann. Math. Logic 9 (1976), 187-222.

3. W. W. Comfort and S. Negrepontis, Chain conditions in topology, Cambridge Tracts in Math., no. 79, Cambridge Univ. Press, 1982.

4. W. Deuber, Partitionstheoreme für Graphen, Math. Helv. 50 (1975), 311-320.

5. K. J. Devlin, Aspects of constructibility, Lecture Notes in Math., vol. 354, Springer-Verlag, Berlin and New York, 1973.

6. P. Erdös, F. Galvin and A. Hajnal, On set-systems having large chromatic number and not containing prescribed subsystems, Infinite and Finite Sets, Colloq. Math. Soc. János. Bolyai, no. 19 (Keszthely, Hungary), 1973, pp. 425-513.

7. P. Erdös and A. Hajnal, On chromatic number of graphs and set-systems, Acta Math. Acad. Sci. Hungar. 17 (1966), 61 -99.

8. __ On decompositions of graphs, Acta Math. Acad. Sci. Hungar. 18 (1967), 359-377.

9. __ On chromatic number of infinite graphs, Theory of Graphs (Proc. Colloq., Tihany, 1966) P. Erdös and G. Katona, eds.), Academic Press, New York, 1968, pp. 83-89.

10. P. Erdös, A. Hajnal and L. Pósa, Strong embedding of graphs into colored graphs, Infinite and Finite Sets (Keszthely, 1973), Colloq. Math. Soc. János Bolyai, no. 10, pp. 585-595. 
11. P. Erdös, A. Hajnal and B. Rothschild, On chromatic number of graphs and set systems, Cambridge Summer School in Mathematical Logic (Cambridge, England, 1971), Lecture Notes in Math., vol. 337, Springer-Verlag and New York, 1973, pp. 531-538.

12. J. Folkman, Graphs with monochromatic complete subgraphs in every edge coloring, SIAM Appl. Math. 18 (1970), 19-24.

13. R. L. Graham, Rudiments of Ramsey theory, C.B.M.S. Regional Conf. Ser. Math, no. 45, Amer. Math. Soc., Providence, R. I., 1981.

14. A. Hajnal and J. Pach, Monochromatic paths in infinite coloured graphs, Finite and Infinite Sets, Colloq. Math. Soc. János Bolyai, no. 37, (Eger, Hungary, 1981), 1984, pp. 359-369.

15. P. Komjáth, A note on Hajnal-Máté graphs, Studia Sci. Math. Hungar. 15 (1980), 275-276.

16. __ The colouring number, Proc. London Math. Soc. 54 (1987), 1-14.

17. P. Komjáth and V. Rödl, Coloring of universal graphs, Graphs and Combinatorics 2 (1986), 55-61.

18. K. Kunen, Set theory, An introduction to independence proofs, North-Holland, 1980.

19. J. Nesetril and V. Rödl, A Ramsey graph without triangles exists for any graph without triangles, Infinite and Finite Sets, Colloq. Math. Soc. János Bolyai, no. 10 (Keszthely, Hungary), 1973, pp. 1127-1132.

20. _ Partitions of vertices, Comment. Math. Univ. Carolin. 17 (1976), 85-95.

21. __ On a probabilistic graph-theoretical method, Proc. Amer. Math. Soc. 72 (1978), 417-421.

22. S. Shelah, Colouring without triangles and partition relations, Israel J. Math. 20 (1975), 1-12.

23. __, Proper forcing, Lecture Notes in Math., vol. 940, Springer-Verlag, Berlin and New York,

Mathematics Institute of the Hungarian ACademy of Science, Budapest POB 127, 1364, HUNGARY

Department of Computer Science, EötVös University, Muzeum KRT. 6-8, BUDAPEST, 1088, HUNGARY 